\title{
Automated Methods for Profiling the Axonal Transport of Secretory BDNF Granules in Live Cultured Neurons from Time-lapse Microscopy Data
} Amit Mukherjee*, Andrew Cohen", Badrinath Roysam* , Brian Jenkins ${ }^{* *}$, Cheng Fang ${ }^{* *}$, Stefanie Kaech Petrie $^{* *}$, and Gary Banker ${ }^{* *}$

*Electrical, Computer \& Systems Engg., Rensselaer Polytechnic Institute, Troy NY 12180, USA

*** Jungers Center for Neurosciences Research, OHSU, Portland, OR 97239, USA

Patterns of axonal transport of brain-derived neurotrophic factor (BDNF) granules are of vital interest to studies of neurodegenerative diseases, especially Huntington's and Alzheimer's ${ }^{1}$. Protocols now exist for time-lapse microscopy of live cultured neurons using a combination of fluorescent protein labeling \& sensitive instrumentatuion ${ }^{2}$. However, the tracking of granules is currently performed manually. This is laborious, time-consuming, and subjective. There is a compelling need for automated computational tools to extract measurements of granule transport accurately, objectively, and rapidly. Recently, Jaqaman et al. ${ }^{3}$, have described robust particle tracking methods for time-lapse sequences. However, these methods are ineffective for tracking BDNF transport due to several factors: their small size, low signal-to-noise, high speed compared to the frame rate and particle size, and frequent crossing of particle trajectories.

This paper presents a novel and effective method to overcome the above limitations using a combination of approaches. An expression construct for mCherry-tagged BDNF was transfected into mouse cortical neurons, derived from E18 embryos and cultured for 7 days ${ }^{4}$. Co-expression of soluble EGFP was used to delineate neuronal morphology and identify areas where the orientation of the axon relative to the cell body was clear and the direction of transport (anterograde/retrograde) was evident. Cells grown on a coverslip were mounted in a closed \& heated chamber and imaged by a Yokogawa CSU-10 spinning disk confocal on a Nikon TE2000 inverted scope with continuous axial-drift compensation. For illumination, an AOTF-controlled laser line selection on a Coherent Innova 70C Krypton Argon Ion laser was used. Images were collected at $\sim 2 \mathrm{~Hz}$ on a high-resolution Hamamatsu Orca ER CCD camera. Fig. 1A shows a closeup from a time-lapse series to illustrate the difficulty of visual tracking. Fig. 1B shows the result of automated axon tracing (blue line) from a user-provided pair of proximal and distal points on an axon. Fig. $1 \mathrm{C}$ is an automatically generated kymograph for the time-lapse BDNF data over the selected axon segment. The red box corresponds to the sample frames in Fig. 1A. Fig. 1D shows the Velogram - the result of directional filtering displayed using the color coding scheme shown by the color bar at the bottom. The ridges of the velogram tracks represent particle trajectories that are automatically traced using a graph-theoretic minimum spanning tree algorithm to generate a large number of track fragments that are subsequently joined using a support vector regression algorithm ${ }^{5}$ that is trained using a set of examples. The end result is a set of particle tracks shown in Fig. 1E from which histograms of particle transport speeds can be derived as shown in Fig. 1F.

\section{References}

[1] G. Lynch et al., Eur J Pharmacol. 585 (2008) 2-13.

[2] C.L. Erica et al., Biochimica et Biophysica Acta. 1762 (2006) 1094-1108.

[3] Jaqaman et al., Nature Methods - 5, 695 - 702 (2008).

[4] S. Kaech et al., Nature Protocols 1, - 2406 - 2415 (2006).

[5] A. J. Smola et al, Statistics and Computing, pp 199-222, 2004.

[6] This work is supported by Cure Huntington's Disease Inc., and NSF Grant EEC9986821. 

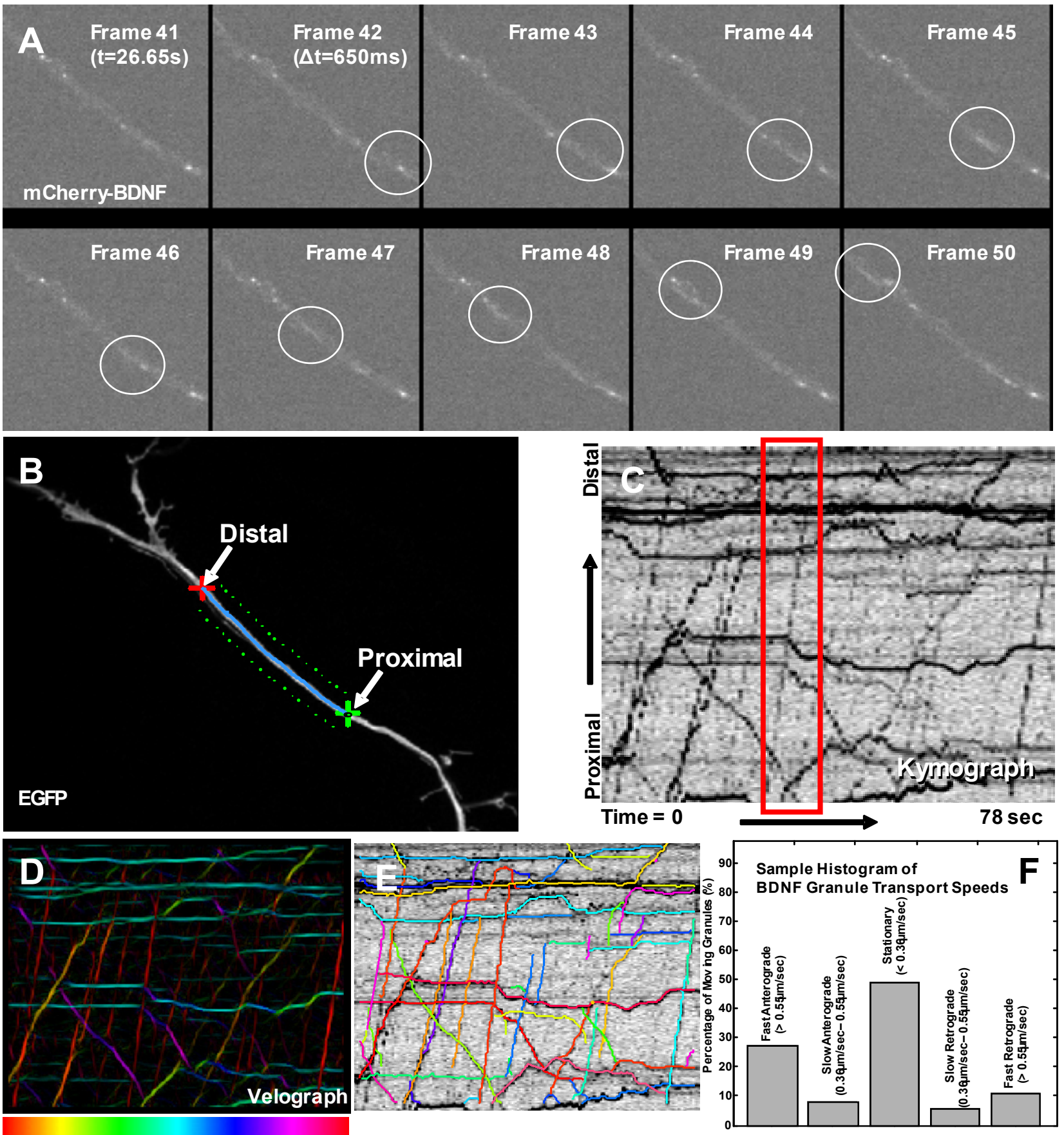

Fast Anterograde Stationary Fast Retrograde

Figure 1: Illustrating the methodology for a sample time-lapse sequence. (A) Sample close-up frames from a time-lapse recording with a very faint BDNF granule indicated by the circles. (B) The section of axon selected for velocity profiling - the distal and proximal points are specified using a computer mouse, and the trace of the axon (blue) is generated automatically. (C) Kymograph for the axon segment for a $78 \mathrm{sec}$ recording, with the trajectory of the granule in Panel A highlighted by the red box. (D) Shows the color-coded Velogram representation, the color bar at the bottom shows the color coding scheme (E) The results of automated tracking of BDNF granules over the $78 \mathrm{sec}$ sequence. (F) The velocity histogram profiling the granules in the timelapse movie. 